\title{
Anderson localization at the subwavelength scale for surface plasmon polaritons in disordered arrays of metallic nanowires
}

\author{
Xianling Shi, ${ }^{1}$ Xianfeng Chen, ${ }^{1}$ Boris A. Malomed, ${ }^{2}$ Nicolae C. Panoiu, ${ }^{3}$ and Fangwei Ye ${ }^{1, *}$ \\ ${ }^{1}$ State Key Laboratory of Advanced Optical Communication Systems and Networks, Department of Physics and Astronomy, Shanghai Jiao \\ Tong University, Shanghai 200240, China \\ ${ }^{2}$ Department of Physical Electronics, School of Electrical Engineering, Faculty of Engineering, Tel Aviv University, Tel Aviv 69978, Israel \\ ${ }^{3}$ Department of Electronic and Electrical Engineering, University College London, Torrington Place, London WC1E 7JE, United Kingdom
}

(Received 7 September 2013; published 20 May 2014)

\begin{abstract}
Using one- and two-dimensional random arrays of coupled metallic nanowires as a generic example of disordered plasmonic systems, we demonstrate that the structural disorder induces localization of light in these nanostructures at a deep-subwavelength scale. The ab initio analysis is based on solving the complete set of three-dimensional Maxwell equations. We find that random variations of the radius of coupled plasmonic nanowires are sufficient to induce the Anderson localization (AL) of surface plasmon polaritons (SPPs), the size of these trapped modes being significantly smaller than the optical wavelength. Remarkably, the optical-gain coefficient, needed to compensate for losses in the plasmonic components of the system, is much smaller than the loss coefficient of the metal, which is obviously beneficial for the realization of the AL in plasmonic nanostructures. The dynamics of excitation and propagation of the Anderson-localized SPPs are addressed too.

DOI: 10.1103/PhysRevB.89.195428

PACS number(s): 78.67.Pt, 42.82.Et, 73.20.Mf, 78.68.+m
\end{abstract}

\section{INTRODUCTION}

When the size of photonic devices is reduced to the subwavelength scale, the confinement and guiding of the electromagnetic energy is severely hampered by the diffraction of optical fields. This limitation represents the main roadblock on the way to the integration of photonic circuits at the nanoscale level [1,2]. An effective way to overcome this limitation is to employ surface plasmon polariton (SPP) waves [3-5], whose strong confinement at the metallic surface and deep-subwavelength characteristic scale make it possible to achieve a strong coupling between the optical fields and nanosized photonic structures. In this context, one of major goals of the work with SPP-based nanodevices is to develop new techniques for precise beam steering, optical switching, and field manipulations at the subwavelength scale. A very promising approach toward this goal is to employ arrays of metallic nanowires, also known as plasmonic crystals [6-12], where the optical coupling of SPPs propagating in adjacent nanowires is controlled by dielectric properties of the embedding medium [13-15]. In particular, the use of periodic arrays of nanowires makes it possible to engineer the effective optical dispersion with an unprecedented degree of flexibility [9-12].

In this context, a natural question is to what extent structural disorder, which is inevitably introduced by nanofabrication, or purposely built into the system, affects the physical properties of the plasmonic crystals and thus limits the functionality of subwavelength plasmonic nanodevices. In particular, it is well known that the structural disorder may profoundly affect the spectrum of wave modes, with the Anderson localization (AL) being, perhaps, the most spectacular effect of that kind. This is a fundamental wave phenomenon, which was first predicted in solid-state physics as the localization of electron wave functions in disordered lattices [16]. It was later established that the AL is a ubiquitous effect that occurs in a multitude

\footnotetext{
*fangweiye@sjtu.edu.cn
}

of settings in which waves interact with disordered potentials, including light [17-19], matter waves [20,21], and sound [22]. Disorder effects are expected to be particularly important at the subwavelength scale, including plasmonic systems similar to those investigated in this work, as the coupling between the waves and the underlying disordered system is enhanced at that scale. In this context, the AL of SPPs was predicted in metal-dielectric percolation composites [23], and the effects of randomly located scatters on SPPs guiding along the surface of gold films were observed experimentally [24].

In this paper, we study the influence of the structural disorder on the spatial distribution of the plasmonic field and its propagation in one- and two-dimensional (1D and 2D) arrays of coupled metallic nanowires. Solving the full system of the corresponding Maxwell equations (MEs), we find that a random distribution of radii of the nanowires leads to transverse spatial localization of collective SPP excitations (plasmonic supermodes of the array). The characteristic spatial confinement of the plasmonic field may be significantly smaller than the optical wavelength, $\lambda$, which demonstrates that plasmonic structures can be employed to implement the subwavelength AL of the electromagnetic field. To facilitate experimental observation of such extreme localization of light, we also study the feasibility of the compensation of optical losses by means of embedded gain elements. Our analysis shows that the deep-subwavelength Anderson-localized SPPs may be maintained at extremely low gain levels, or even without gain.

\section{PHYSICAL SYSTEM AND THEORETICAL MODEL}

We start by considering $1 \mathrm{D}$ arrays of $N$ coupled metallic nanowires, which are oriented along the $z$ axis, being equally spaced (center-to-center) in the transverse direction, $x$, by distance $d$; see Fig. 1(a). The structural disorder is introduced by fixing radii of the nanowires in the array, with discrete coordinate $n$, as $a_{n}=a+\delta_{n}$, where $a$ is the average radius (we take $a=40 \mathrm{~nm}$ ), and $\delta_{n}$ is a random deviation. We assume that 
(a)

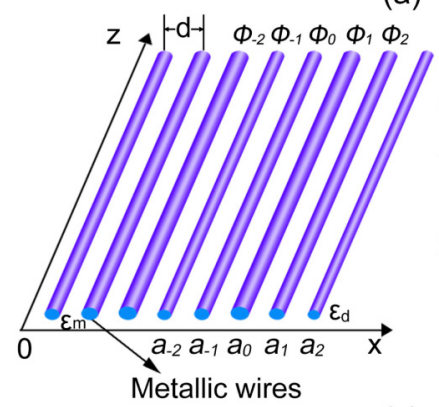

(c)

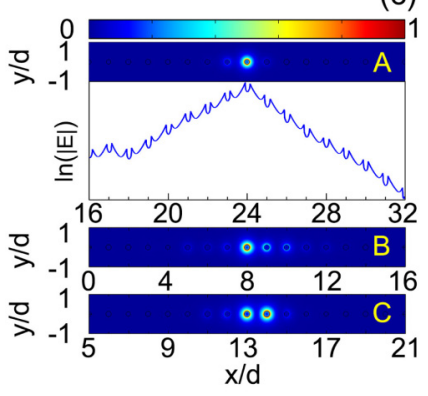

(b)

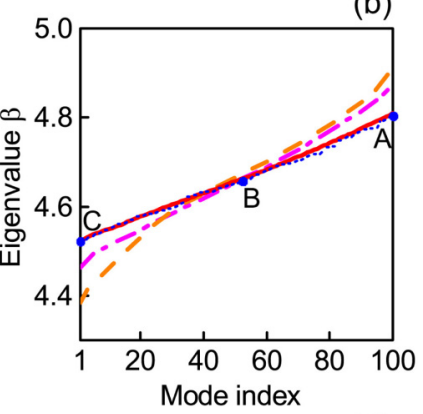

(d)

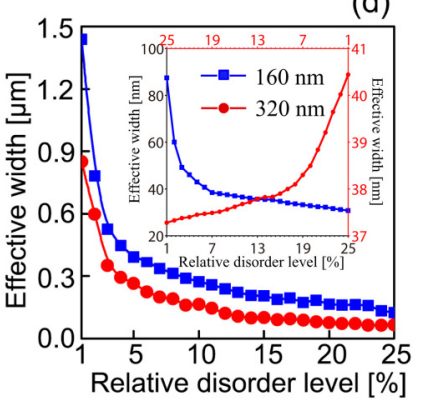

FIG. 1. (Color online) (a) A disordered plasmonic array. (b) The spectrum of supermodes of the array, averaged over an ensemble of 100 randomness realizations, produced by the CMT equations (1) in the nearest-neighbor (dot-dashed line) and next-nearest-neighbor (dashed line) approximations, as well as obtained from the full ME set (solid line). The dotted line stands for the spectrum corresponding to one particular realization of the randomness. Labels $A-C$ indicate the location of the ALMs in (c). (c) Generic examples of electric-field intensity profiles of ALMs in the array. The logarithmic plot shows the transverse profile of mode $(A)$ at $y=0$. In (b) and (c), the array's spacing is $d=8 a$, and the randomness strength is $\Delta=10 \%$ (d). The effective width of staggered ALMs vs $\Delta$, as calculated with $d=4 a$ and $8 a$, for an ensemble of 200 randomly composed plasmonic arrays. Other parameters in (b) $-(\mathrm{d})$ are $\lambda=1.55 \mu \mathrm{m}, a=40 \mathrm{~nm}$, and $N=$ 100. Inset of (d) plots the effective width of ALMs at $\lambda=0.6328 \mu \mathrm{m}$.

$\delta_{n}$ is uniformly distributed in the interval of $[-\delta, \delta]$, with $\delta<a$, the level of the disorder being characterized by $\Delta \equiv \delta / a$. The equal spacing between nanowires makes the present setting different from fully random plasmonic structures, e.g., planar randomly distributed metallic scatterers with random sizes [23,24]; actually, the nature of the disorder in the present system is similar to that introduced by Anderson in his seminal work [16].

Previous studies of the AL in systems of coupled waveguides were based on the paraxial approximation for the propagation of electromagnetic waves, chiefly because the relative variation of the refractive index in such systems is small, hence the characteristic scale of the AL of light is much larger than $\lambda$. However, the paraxial approximation is not valid for plasmonic systems, where the relative variation of the refractive index is large by definition, allowing, as we show below, the AL scale to be significantly smaller than $\lambda$. This fact implies that the use of the full system of three-dimensional (3D) MEs is necessary. Thus, our analysis starts ab initio, solving the 3D ME system in the framework of the COMSOL shell [25]. In the simulations, a predefined triangular fine

mesh with a maximum-size element of $10 \mathrm{~nm}$ was used. The resulting face mesh sweeps along the propagation direction of the nanowires with a step of $500 \mathrm{~nm}$. Appropriate scattering boundary conditions were used to mimic open boundaries. A convergence analysis was conducted to ensure that the results vary within tolerable errors.

We set the permittivity of the dielectric background material to be $\epsilon_{d}=12.25$, which corresponds, e.g., to $\mathrm{Si}$ or GaAs, and we use the Drude model to describe the permittivity of the metal, $\epsilon_{m}=1-\omega_{p}^{2} /[\omega(\omega+i \nu)]$. We assume that the nanowires are made of silver, with plasmon and damping frequencies $\omega_{p}=13.7 \times 10^{15} \mathrm{rad} \mathrm{s}^{-1}$ and $v=$ $2.7 \times 10^{14} \mathrm{rad} \mathrm{s}^{-1}[26]$.

To gain better insight into the physics of the SPP localization, we compared results produced by the ME system with those obtained from the paraxial model for the propagation of SPPs in the disordered plasmonic arrays, based on the coupled-mode theory (CMT). A detailed derivation of the CMT model $[13,27,28]$ leads to the discrete Schrödinger equation with a long-range coupling:

$$
i \frac{d \phi_{n}}{d z}+b_{n} \phi_{n}+\sum_{j \geqslant 1} \kappa_{j}\left(\phi_{n-j}+\phi_{n+j}\right)=0,
$$

where $b_{n}$ is the propagation constant of the mode associated with the $n$th nanowire. Apart from the $z$-dependent phase, the nonvanishing field components of this mode, $\mathbf{e}_{r}, \mathbf{e}_{z}$, and $\mathbf{h}_{\phi}$, depend only on the radial coordinate, $r_{\perp}$. In Eq. (1), $\kappa_{j}$ is the coupling coefficient between nanowires separated by discrete distance $j$, which can be calculated using fields of the plasmon mode of the nanowire, $\mathbf{e}\left(r_{\perp}\right)$ and $\mathbf{h}\left(r_{\perp}\right)$, and the distribution of the dielectric constant in the plasmonic system, $\epsilon\left(\mathbf{r}_{\perp}\right)$ [13]. The corresponding modal fields are $\mathbf{E}(\mathbf{r})=\sum_{n} \mathbf{e}_{n}\left(r_{\perp}\right) e^{i\left(\beta k_{0} z-\omega t\right)}$ and $\mathbf{H}(\mathbf{r})=\sum_{n} \mathbf{h}_{n}\left(r_{\perp}\right) e^{i\left(\beta k_{0} z-\omega t\right)}$, where $\beta$ is the effective refractive index of the plasmon mode, and $k_{0}=\omega / c$ is the wave number in vacuum at carrier frequency $\omega$.

\section{RESULTS AND DISCUSSION}

Results typical for the disordered plasmonic arrays are shown in Figs. 1(b) and 1(c), where the spectrum of the supermodes (the transmission band) and several representative modal field profiles of AL modes (ALMs) are displayed, respectively. Here we only show results for the first transmission band, as higher-order ones do not support localized eigenmodes. Our simulations, based on both the CMT and full ME, reveal that, as expected, for small disorder $(\Delta \lesssim 5 \%)$ all generic supermodes of the finite plasmonic array feature extended profiles, i.e., the AL does not occur, as for such a weak disorder, the localization length may be larger than the system's size (for some particular realizations of the disorder, the $\mathrm{AL}$ does occur even if $\Delta$ is as small as $1 \%$; however, for the very weak disorder, the AL is not a generic feature of the plasmonic field). When the disorder strength exceeds $\Delta \simeq 5 \%$, two strongly localized modes emerge at edges of the transmission band, as shown in Fig. 1(c). As may be naturally expected, ALMs near the bottom of the band are unstaggered, in the sense that the phase of the longitudinal component of the electric field, $E_{z}$, is constant across the array, while the ALMs at the top of the band are staggered $\left(E_{z}\right.$ in adjacent nanowires 
points in opposite directions). ALMs located in the central part of the band feature a mixed structure, with parts of the mode staggered and other parts unstaggered. When $\Delta$ increases further, additional supermodes become more localized and evolve into ALMs. For all values of $\Delta$ at which the AL occurs, the ALMs at the edges of the band are, typically, localized much stronger than near its center.

Surprisingly, the CMT equations provide a somewhat more accurate description of the plasmonic supermodes when only the nearest-neighbor coupling is kept in Eq. (1). Nevertheless, a particularly large discrepancy between the predictions of the CMT and 3D ME is observed for the modes at the edges of the transmission band. The ME and CMT not only predict significantly different values for the propagation constant of the supermodes of the plasmonic array, but also the field profiles of the supermodes produced by these two methods are in poor agreement (not shown here). These finding clearly demonstrate the necessity of the use of the 3D ME for modeling strongly coupled, high-index-contrast systems, such as our plasmonic arrays, as the CMT yields a coarse approximation in this setting.

Figure 1(d) presents the effective width of the ALMs, defined as

$$
w_{\text {eff }}=\left\langle\left[\frac{\int_{-\infty}^{\infty}|\mathbf{E}(x, y=0)|^{2}\left(x-x_{0}\right)^{2} d x}{\int_{-\infty}^{\infty}|\mathbf{E}(x, y=0)|^{2} d x}\right]^{\frac{1}{2}}\right\rangle,
$$

where $x_{0} \equiv \int_{-\infty}^{+\infty}|\mathbf{E}(x, y=0)|^{2} x d x / \int_{-\infty}^{+\infty}|\mathbf{E}(x, y=0)|^{2} d x$ is the central coordinate of the mode, \langle\rangle stands for averaging over multiple realizations of the randomness with the same degree of disorder, and the electric field is obtained by solving the 3D ME. Naturally, the width decreases with the increase of the randomness strength, asymptotically reaching a constant value for high disorder levels. When this minimum width is reached, the plasmonic field is localized around a single nanowire. It is worth noting that the width can become much smaller than the wavelength even at rather low disorder levels. For a given randomness strength, the modal width increases with the decrease of the separation between the nanowires, because smaller spacing leads to stronger coupling between them, making stronger randomness necessary to induce the AL.

An important result inferred from Figs. 1(b) and 1(d) is that statistical averaging over the ensemble of disordered arrays converges rather fast, hence a relatively small number of arrays with different realizations of the disorder need to be actually considered, deviations between the results produced by particular realizations being small. This observation significantly reduces the required computational time and thus greatly simplifies the analysis. This result is explained by a weak dependence of the mode's propagation constant and coupling strength on the radius of the nanowires. Equally important is the potential implication of this result for the design of AL-based plasmonic nanodevices, as one may expect that their properties depend weakly on the particular realization of the system randomness [29].

The Ohmic loss in metallic nanowires causes decay of propagating ALMs, which can make their observation a challenging task. A promising scheme to offset the loss is to embed the array into a dielectric medium carrying optical gain, provided, e.g., by pumped quantum dots or wells [30].

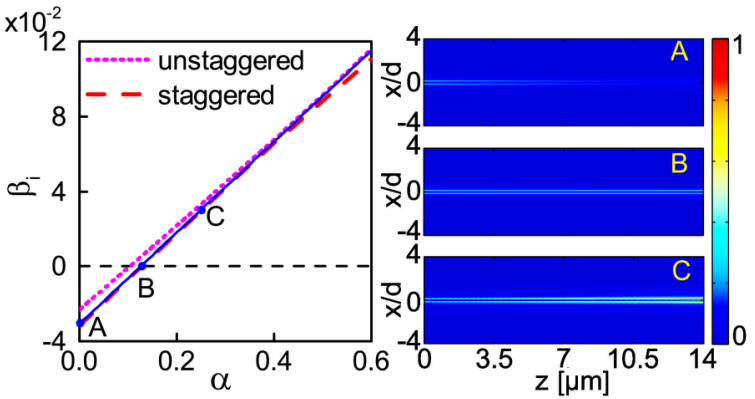

FIG. 2. (Color online) Left panel: the imaginary part of the propagation constant, $\beta_{i}$, vs the gain of the host dielectric material, $\alpha$, for staggered and unstaggered ALMs, averaged over 100 randomness realizations. The permittivities of the host material and metal are, respectively, $\epsilon_{d}=12.25+i \alpha$ and $\epsilon_{m}=-125.46-2.84 i$ (at $\lambda=$ $1.55 \mu \mathrm{m})$. Right panels: the propagation of the staggered ALM at three different values of the gain for a specific randomness realization (the solid line in the left panel): $\alpha=0$ (a), $\alpha=\alpha_{\mathrm{cr}}=0.126$ (b), and $\alpha=0.25$ (c). The parameters are $d=320 \mathrm{~nm}, N=20$, and $\Delta=15 \%$.

Figure 2 summarizes results produced by solving the 3D ME for the loss characteristics of ALMs in the present setting, as well as their formation and propagation in the presence of the gain. In the simulations, we assumed that the metal's permittivity is $\epsilon_{m}=-125.46-2.84 i$, which corresponds to silver at $1550 \mathrm{~nm}$, and the permittivity of the embedding medium is $\epsilon_{d}=12.25+\alpha i$, where $\alpha$ is the gain coefficient.

The most relevant quantity in this context is the imaginary part of the ensemble-averaged modal propagation constant, $\beta_{i}$, as it directly determines the loss. The dependence of $\beta_{i}$ of the staggered and unstaggered ALMs on $\alpha$ is displayed in Fig. 2. Two significant features are revealed by this figure. First, dependence $\beta_{i}(\alpha)$ is almost linear. The reason for this is that the gain/loss part of the permittivity of the metal and the gain medium is much smaller than the corresponding real part (especially in the metal, as stated above), which means that the field profile of the ALMs remains almost unchanged as one varies $\alpha$ (our numerical results directly confirm a weak dependence of the modal profile on $\alpha$ ). As the effective loss of the mode is given by a certain spatially weighted average of the imaginary part of the permittivity over the nearly constant modal field profile, this indeed implies that the corresponding loss coefficient depends on the gain/loss almost linearly. Second, the gain coefficient, $\alpha_{\mathrm{cr}}$, at which the loss is compensated, differs significantly from the loss coefficient of the metal, being, quite surprisingly, much smaller. This is explained by the fact that the mode does not distribute its field evenly between the metallic (lossy) and gain regions. More specifically, the simulations show that $\beta_{i}=0$ is achieved at $\alpha_{\mathrm{cr}}=0.126 \ll 2.84$, which is more than 20 times smaller than the loss coefficient. These findings are also illustrated by the propagation patterns of ALMs, shown in the right panel of Fig. 2 for three different values of the gain coefficient: $\alpha=0<\alpha_{\mathrm{cr}}, \alpha=\alpha_{\mathrm{cr}}$, and $\alpha=0.25>\alpha_{\mathrm{cr}}$.

In addition to the gain compensation, the excitation of ALMs, provided by an appropriate input coupled into the system, is also an issue of critical significance. Essential 


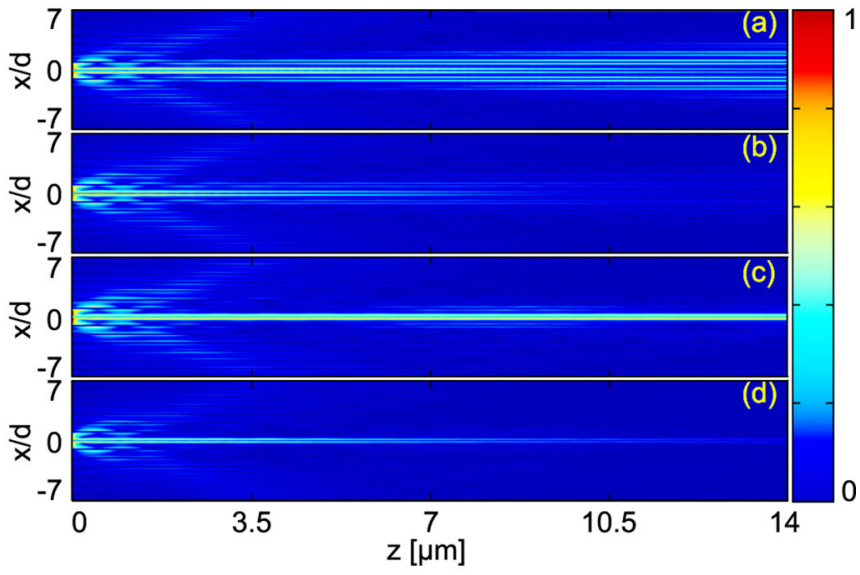

FIG. 3. (Color online) The propagation of a modulated Gaussian with optimized parameters, defined by the following input: $E_{x}(x, y)=$ $\sin \left[2 \pi\left(x-x_{0}\right) / d\right] \exp \left[-\left(x-x_{0}\right)^{2} / w^{2}\right] \exp \left(-y^{2} / w^{2}\right)$, which implies the excitation of a staggered ALM. Panels (a) and (b) correspond to the periodic arrays, whereas (c) and (d) pertain to the randomized ones. Metallic loss is not incorporated in (a) and (c), but is included in (b) and (d). The parameters are $\lambda=1.55 \mu \mathrm{m}, d=320 \mathrm{~nm}, x_{0}=d / 2$, $w=d / 2, N=20$, and $\Delta=15 \%$.

findings pertaining to this issue are summarized in Fig. 3, which displays the excitation of ALMs by a modulated Gaussian beam, whose initial width and input location in the array are optimized using the field profile of the eigenmode provided by the above analysis. The objective is to achieve the shortest ALM formation length, defined as the distance required for the input beam to reshape itself into an ALM. For the ALM to be observable in the setup in which the loss is not compensated by the gain, the formation length should be shorter than the characteristic modal decay length. Simulations presented in Figs. 3(c) and 3(d) clearly demonstrate that the Gaussian, coupled into the disordered plasmonic array, evolves into an ALM after passing just a few microns, a part of the input energy being shed off in the form of radiation waves. In the course of the evolution, the beam preserves its width, although its intensity exhibits an overall decrease if realistic loss is included; see Fig. 3(d). By contrast, significant beam diffraction is observed, over the entire propagation distance, in arrays with vanishing randomness, as seen in Figs. 3(a) and 3(b). Thus, ALMs may be observed even in the absence of the compensating gain, provided that the input profile is properly adjusted. On the other hand, if the input deviates significantly from the optimized shape, one should add the gain to make the ALM formation length smaller than the modal decay length.

The localization of SPPs is also possible in 2D disordered nanowire arrays. The main features of the respective phenomenology are similar to those reported above for the 1D setting, therefore we only briefly present them here. Two representative examples of 2D ALMs are shown in Fig. 4, where deep subwavelength confinement of the plasmonic field, in both transverse directions, is clearly observed. In one case, the ALM is formed inside the array, therefore we name it a bulk mode, whereas the other one is located at the boundary of the array, and may be considered as a surface ALM. In both cases, two ALMs formed under different randomness realizations are displayed, which again shows that
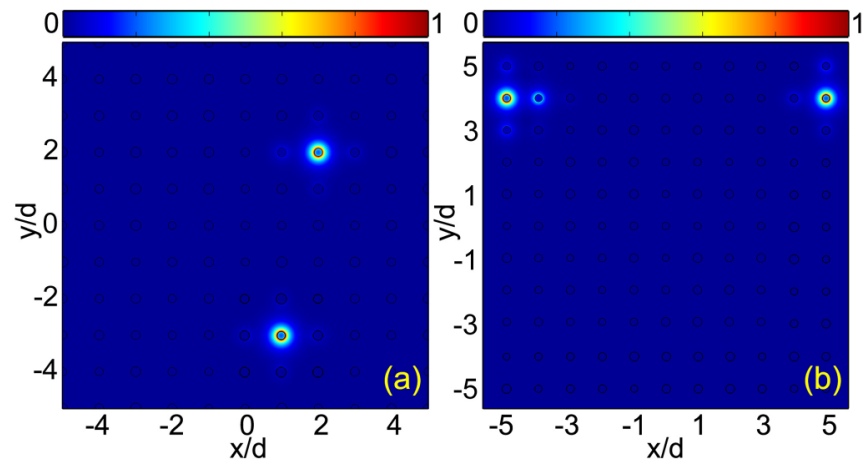

FIG. 4. (Color online) Examples of ALMs formed in a 2D disordered plasmonic array. (a) and (b) Intensity of the electric field in bulk and surface ALMs, respectively. In each panel, two modes are displayed, corresponding to different realizations of the randomness. Parameters are $d=320 \mathrm{~nm}, \Delta=15 \%$, and $\lambda=1.55 \mu \mathrm{m}$.

the characteristics of AL in our disordered system depend weakly on the particular realization. In particular, it is observed that, even for a relatively weak disorder, the field is almost entirely confined around a single nanowire. Our computations of the propagation constant of the supermodes show that the predictions of the CMT are still less accurate in two dimensions than in one dimension.

\section{CONCLUSIONS}

In conclusion, by solving the complete set of the 3D Maxwell equations, we have demonstrated that the AL of SPPs can be achieved in 1D and 2D arrays of metallic nanowires with a varying degree of the structural disorder. The characteristic localization length of these plasmonic ALMs may be much smaller than the optical wavelength. We have investigated the influence of the metallic loss and gain of the host medium on the plasmonic ALMs, concluding that the loss is compensated by the gain whose strength is much smaller than the loss rate of the metallic component of the plasmonic array. These results suggest that experimental observation of the ALMs is possible with the currently available nanofabrication and experimental techniques.

It is worth noting that the proposed settings can be readily extended to the midinfrared and $\mathrm{THz}$ spectral regions by using other plasmonic systems, such as arrays of graphene ribbons [31-33]. We also point out that our approach, based on the full ME system, may be applied as well to the subwavelength localization of atomic excitations, a phenomenon that has been recently observed experimentally [34].

\section{ACKNOWLEDGMENTS}

The authors thank D. Mihalache and S. K. Turitsyn for useful discussions. F.Y. acknowledges financial support from the National Natural Scientific Funding of China (NSFC) (Grant No. 11104181) and the Innovation Program of Shanghai Municipal Education Commission (Grant No. 13ZZ022). X.C. acknowledges financial support from NSFC (Grant No. 61125503). The work of N.C.P. was supported by the Engineering and Physical Sciences Research Council (Grant No. EP/J018473/1). 
[1] S. A. Maier, Plasmonics: Fundamentals and Applications (Springer, Berlin, 2007).

[2] W. Barnes, A. Dereux, and T. Ebbesen, Nature (London) 424, 824 (2003).

[3] H. Raether, Surface Plasmons on Smooth and Rough Surfaces and on Gratings, Springer Tracts in Modern Physics Vol. 111 (Springer, Berlin, 1988).

[4] A. V. Zayats, I. I. Smolyaninov, and A. A. Maradudin, Phys. Rep. 408, 131 (2005).

[5] E. Ozbay, Science 311, 189 (2006).

[6] S. I. Bozhevolnyi, J. Erland, K. Leosson, P. M. W. Skovgaard, and J. M. Hvam, Phys. Rev. Lett. 86, 3008 (2001).

[7] T. A. Kelf, Y. Sugawara, J. J. Baumberg, M. Abdelsalam, and P. N. Bartlett, Phys. Rev. Lett. 95, 116802 (2005).

[8] A. R. Tao, D. P. Ceperley, P. Sinsermsuksakul, A. R. Neureuther, and P. Yang, Nano Lett. 8, 4033 (2008).

[9] B. Vasić and R. Gajić, J. Opt. Soc. Am. B 29, 2964 (2012).

[10] C. Bauer, G. Kobiela, and H. Giessen, Sci. Rep. 2, 681 (2012).

[11] A. E. Miroshnichenko, B. Lukyanchuk, S. A. Maier, and Y. S. Kivshar, ACS Nano 6, 837 (2012).

[12] I. V. Iorsh, P. A. Belov, A. A. Zharov, I. V. Shadrivov, and Y. S. Kivshar, Phys. Rev. A 86, 023819 (2012).

[13] F. Ye, D. Mihalache, B. Hu, and N. C. Panoiu, Phys. Rev. Lett. 104, 106802 (2010)

[14] F. Ye, D. Mihalache, B. Hu, and N. C. Panoiu, Opt. Lett. 36, 1179 (2011).

[15] Y. Kou, F. Ye, and X. Chen, Opt. Lett. 38, 1271 (2013).

[16] P. W. Anderson, Phys. Rev. 109, 1492 (1958).

[17] S. John, Phys. Rev. Lett. 53, 2169 (1984).

[18] T. Schwartz, G. Bartal, S. Fishman, and M. Segev, Nature (London) 446, 52 (2007).

[19] Y. Lahini, A. Avidan, F. Pozzi, M. Sorel, R. Morandotti, D. N. Christodoulides, and Y. Silberberg, Phys. Rev. Lett. 100, 013906 (2008).

[20] J. Billy, V. Josse, Z. Zuo, A. Bernard, B. Hambrecht, P. Lugan, D. Clement, L. Sanchez-Palencia, P. Bouyer, and A. Aspect, Nature (London) 453, 891 (2008).
[21] G. Roati, C. D'Errico, L. Fallani, M. Fattori, C. Fort, M. Zaccanti, G. Modugno, M. Modugno, and M. Inguscio, Nature (London) 453, 895 (2008).

[22] H. Hu, A. Strybulevych, J. H. Page, S. E. Skipetrov, and B. A. Van Tiggelen, Nat. Phys. 4, 945 (2008).

[23] A. K. Sarychev, V. A. Shubin, and V. M. Shalaev, Phys. Rev. B 60, 16389 (1999).

[24] S. I. Bozhevolnyi, V. S. Volkov, and K. Leosson, Phys. Rev. Lett. 89, 186801 (2002)

[25] COMSOL MULTIPHYSICS ${ }^{\circledR}$ (http://www.comsol.com).

[26] M. A. Ordal, R. J. Bell, J. R. W. Alexander, L. L. Long, and M. R. Querry, Appl. Opt. 24, 4493 (1985).

[27] F. Ye, D. Mihalache, and N. C. Panoiu, in Spontaneous Symmetry Breaking, Self-Trapping, and Josephson Oscillations, edited by B. A. Malomed, Progress in Optical Science and Photonics Vol. 1 (Springer, Berlin, 2013), pp. 357-375.

[28] See Supplemental Material at http://link.aps.org/supplemental/ 10.1103/PhysRevB.89.195428 for a detailed derivation of the coupled-mode equations.

[29] S. Karbasi, C. R. Mirr, P. G. Yarandi, R. J. Frazier, K. W. Koch, and A. Mafi, Opt. Lett. 37, 2304 (2012).

[30] R. D. Schaller, M. A. Petruska, and V. I. Klimov, J. Phys. Chem. B 107, 13765 (2003).

[31] J. Chen, M. Badioli, P. Alonso-Gonzalez, S. Thongrattanasiri, F. Huth, J. Osmond, M. Spasenovic, A. Centeno, A. Pesquera, P. Godignon, A. Z. Elorza, N. Camara, F. J. G. de Abajo, R. Hillenbrand, and F. H. L. Koppens, Nature (London) 487, 77 (2012).

[32] Z. Fei, A. S. Rodin, G. O. Andreev, W. Bao, A. S. McLeod, M. Wagner, L. M. Zhang, Z. Zhao, M. Thiemens, G. Dominguez, M. M. Fogler, A. H. C. Neto, C. N. Lau, F. Keilmann, and D. N. Basov, Nature (London) 487, 82 (2012).

[33] J. Christensen, A. Manjavacas, S. Thongrattanasiri, F. H. L. Koppens, and F. J. G. de Abajo, ACS Nano 6, 431 (2012).

[34] J. A. Miles, Z. J. Simmons, and D. D. Yavuz, Phys. Rev. X 3, 031014 (2013). 